\title{
Loss of TFF1 promotes Helicobacter pylori-induced $\beta$-catenin activation and gastric tumorigenesis
}

\author{
Mohammed Soutto1,2, Judith Romero-Gallo3, Uma Krishna ${ }^{3}$, M. Blanca Piazuelo ${ }^{3}$, \\ M. Kay Washington ${ }^{4}$, Abbes Belkhiri', Richard M. Peek Jr3,5, Wael El-Rifai ${ }^{1,2,5}$ \\ ${ }^{1}$ Department of Veterans Affairs, Tennessee Valley Healthcare System, Nashville, Tennessee, USA \\ ${ }^{2}$ Department of Surgery, Vanderbilt University Medical Center, Nashville, Tennessee, USA \\ ${ }^{3}$ Division of Gastroenterology, Hepatology, \& Nutrition, Vanderbilt University Medical Center, Nashville, Tennessee, USA \\ ${ }^{4}$ Department of Pathology, Microbiology and Immunology, Vanderbilt University Medical Center, Nashville, Tennessee, USA \\ ${ }^{5}$ Department of Cancer Biology, Vanderbilt University Medical Center, Nashville, Tennessee, USA \\ Correspondence to: \\ Wael El-Rifai, e-mail: wael.el-rifai@vanderbilt.edu
}

Keywords: TFFl, Helicobacter pylori, B-catenin, gastric cancer

Abbreviations: TFFl; trefoil factor 1, H. pylori; Helicobacter pylori, knockout; KO, TCF/LEF; T cell factor/lymphoid

Received: February 18, 2015

Accepted: April 14, 2015

Published: April 27, 2015

\section{ABSTRACT}

Using in vitro and in vivo models, we investigated the role of TFF1 in suppressing $H$. pylori-mediated activation of oncogenic $\beta$-catenin in gastric tumorigenesis. A reconstitution of TFF1 expression in gastric cancer cells decreased $\mathrm{H}$. pylori-induced $\beta$-catenin nuclear translocation, as compared to control $(p<0.001)$. These cells exhibited significantly lower $\beta$-catenin transcriptional activity, measured by pTopFlash reporter, and induction of its target genes (CCND1 and C-MYC), as compared to control. Because of the role of AKT in regulating $\beta$-catenin, we performed Western blot analysis and demonstrated that TFF1 reconstitution abrogates $H$. pylori-induced $p$-AKT (Ser473), $p$ - $\beta$-catenin (Ser552), c-MYC, and CCND1 protein levels. For in vivo validation, we utilized the Tff1-KO gastric neoplasm mouse model. Following infection with PMSS1 $\mathrm{H}$. pylori strain, we detected an increase in the nuclear staining for $\beta$-catenin and Ki-67 with a significant induction in the levels of Ccnd1 and c-Myc in the stomach of the Tff1-KO, as compared to Tff1-WT mice $(p<0.05)$. Only $10 \%$ of uninfected Tff1-KO mice, as opposed to one-third of $\mathrm{H}$. pylori-infected Tff1-KO mice, developed invasive adenocarcinoma $(p=0.03)$. These findings suggest that loss of TFF1 could be a critical step in promoting the $H$. pylori-mediated oncogenic activation of $\beta$-catenin and gastric tumorigenesis.

\section{INTRODUCTION}

Protection of the gastrointestinal tissue integrity is physiologically critical in the presence of persistent irritations caused by microbial flora and harmful agents. The repair of the gastric epithelial cells layer is mediated by several factors that comprise the trefoil factor protein 1 (TFF1) [1]. TFF1 interacts with mucin proteins to stabilize the mucus gel layers of the gastric epithelium and plays a dynamic role in the protection and regeneration of the gastric mucosa [2]. Several reports have demonstrated that $T F F 1$ functions as a gastric tumor suppressor gene $[3,4]$, and loss of Tff1 leads to development of dysplastic lesions and adenocarcinoma in antropyloric gastric tissues in mice [5-7]. In human, TFF1 expression is downregulated in approximately two-thirds of gastric adenocarcinoma due to deletion, loss of heterozygosity, hypermethylation, or transcription regulation [8-12]. A large study of approximately 300 gastric cancers has shown that TFF1 expression is significantly more reduced in cases with differentiated type and intestinal type gastric cancer [12]. This study has shown that a combination of reduced TFF1 expression and high TFF3 expression ( $p=0.018)$ was determined as an independent prognostic factor significantly associated with poor OS in patients with early stage gastric cancer [12]. 
$\beta$-catenin signaling pathway has a well-established role in the regulation of cell growth and proliferation, and its constitutive oncogenic activation is frequent in human cancers, including gastric cancer $[13,14]$. Several reports have shown that $\beta$-catenin activation occurs through its translocation to the nucleus; this process results in its binding to members of the TCF/LEF family of transcription factors [15]. The TCF/ $\beta$-catenin complex activates target genes such as $c-M Y C$ and cyclin D1 $(C C N D 1)$. Inhibition of $\beta$-catenin results in a decreased expression of its target genes and suppression of cell proliferation [15-17].

Helicobacter pylori, a gram-negative bacterium, was first discovered in 1983 by Marshall BJ and Warren JR [18]. The World Health Organization concluded that $H$. pylori is a definite carcinogen based on epidemiological evidence, and it is present in the stomach of approximately half of the world's population $[19,20]$. $H$. pylori infection induces a sequence of events that begins with superficial gastritis, progressing towards chronic atrophic gastritis, intestinal metaplasia, dysplasia, and finally gastric cancer [21]. The cag pathogenicity island (cag-PAI) is the most characterized $H$. pylori virulence factor. CagA (cytotoxin-associated gene) is one of the 30 genes encoded by cag-PAI [22]. Several reports have investigated how CagA controls cellular effects through regulation of specific signal transduction pathways [23]. Among the intracellular signaling affected by CagA is $\beta$-catenin pathway [24-27].

In this study, we investigated the role of TFF 1 in suppressing $H$. pylori-induced activation of $\beta$-catenin using in vitro and in vivo gastric neoplasm models. Our results suggest that TFF1 plays a critical protective role against $H$. pylori-mediated activation of oncogenic $\beta$-catenin where loss of TFF1 could be an important turning point in gastric tumorigenesis.

\section{RESULTS}

\section{Reconstitution of TFF1 decreases $H$. pylori- induced nuclear localization and transcriptional activation of $\beta$-catenin}

In order to determine whether TFF1 reconstitution affects $H$. pylori-mediated activation of $\beta$-catenin, we performed immunofluorescence analysis and utilized two CagA $+H$. pylori carcinogenic strains, J166 and 7.13. Using an adenovirus expression system, we transiently expressed TFF1 or control in MKN28 cells. After $48 \mathrm{~h}$, the cells were infected with $H$. pylori J166 or 7.13 strains for $3 \mathrm{~h}$. In MKN28 control cells, $H$. pylori infection induced a significant increase in $\beta$-catenin nuclear staining as compared to uninfected cells (Figure 1A-1D). However, the reconstitution of TFF1 expression significantly abrogated $H$. pylori- induced $\beta$-catenin nuclear translocation as compared to control cells $(p<0.001$, Figure 1B-1D). In uninfected MKN28 control cells with $\beta$-catenin constitutively activated and localized in the nucleus, the reconstitution of TFF1 expression in these cells led to a nuclear decrease and a membrane increase of $\beta$-catenin staining (Figure 1A-1D). To investigate the suppressive effect of TFF 1 on $H$. pylori-mediated $\beta$-catenin activation, we examined the TCF/LEF transcriptional activation using pTopFlash luciferase reporter. In H. pylori infected cells, TFF1 reconstitution significantly reduced the pTopFlash reporter activity as compared to the controls in J166 $(p<0.05)$ and $7.13(p<0.01)$ strains (Figure 2A). However, in control cells, infection with $H$. pylori without TFF1 expression induced a significant increase of pTopFlash reporter activity as compared to uninfected cells (Figure 2A, $p<0.01$ ). Together, the immunofluorescence and reporter assays data clearly indicated that TFF1 expression decreases $H$. pylori-mediated $\beta$-catenin nuclear localization and transcriptional activation in gastric cancer cells.

\section{TFF1 abrogates $H$. pylori-induced mRNA and protein expression of $\beta$-catenin target genes}

To investigate whether TFF1-dependent suppression of $H$. pylori-induced transcriptional activation of $\beta$-catenin leads to downregulation of $\beta$-catenin downstream targets, we assessed mRNA and protein expression of cyclin $\mathrm{D} 1$ and c-Myc in an in vitro MKN28 cancer cell model. After infection with $H$. pylori strains, qRT-PCR results indicated a significant decrease of mRNA expression of CCND1 (Figure 2B, $p<0.001$ for both $\mathrm{J} 166$ and 7.13 strains) and $c-M Y C$ (Figure $2 \mathrm{C}, p<0.01$ and $p<0.001$ for $\mathrm{J} 166$ and 7.13 strains, respectively) in TFF1 expressing cells as compared to control cells. In addition, $H$. pylori infection of control cells significantly increased CCND1 and $c-M Y C$ mRNA levels as compared to uninfected cells (Figure 2B-2C). In accordance with mRNA results and after infection with H. pylori strains, J166 and 7.13, Western blot analysis demonstrated a decrease of phospho$\beta$-catenin (S552), $\beta$-catenin, c-Myc and cyclin D1 protein levels in MKN28 cells expressing TFF1 as compared to control cells (Figure 3A). We next investigated whether the reconstitution of TFF 1 expression abrogates $H$. pyloriinduced activation of AKT, a major regulator of $\beta$-catenin pathway. Our data indicated that after infection with both strains of H. pylori (J166 and 7.13), phospho-AKT (Ser473) and AKT protein levels were decreased in TFF1 expressing cells as compared to control cells (Figure 3B). Furthermore, the data showed that $H$. pylori infection of control cells increased expression of all analyzed proteins as compared to uninfected cells (Figure 3A-3B). Collectively, the data demonstrated that TFF1 negatively regulates $H$. pyloriinduced $\beta$-catenin activation of target genes in vitro. 

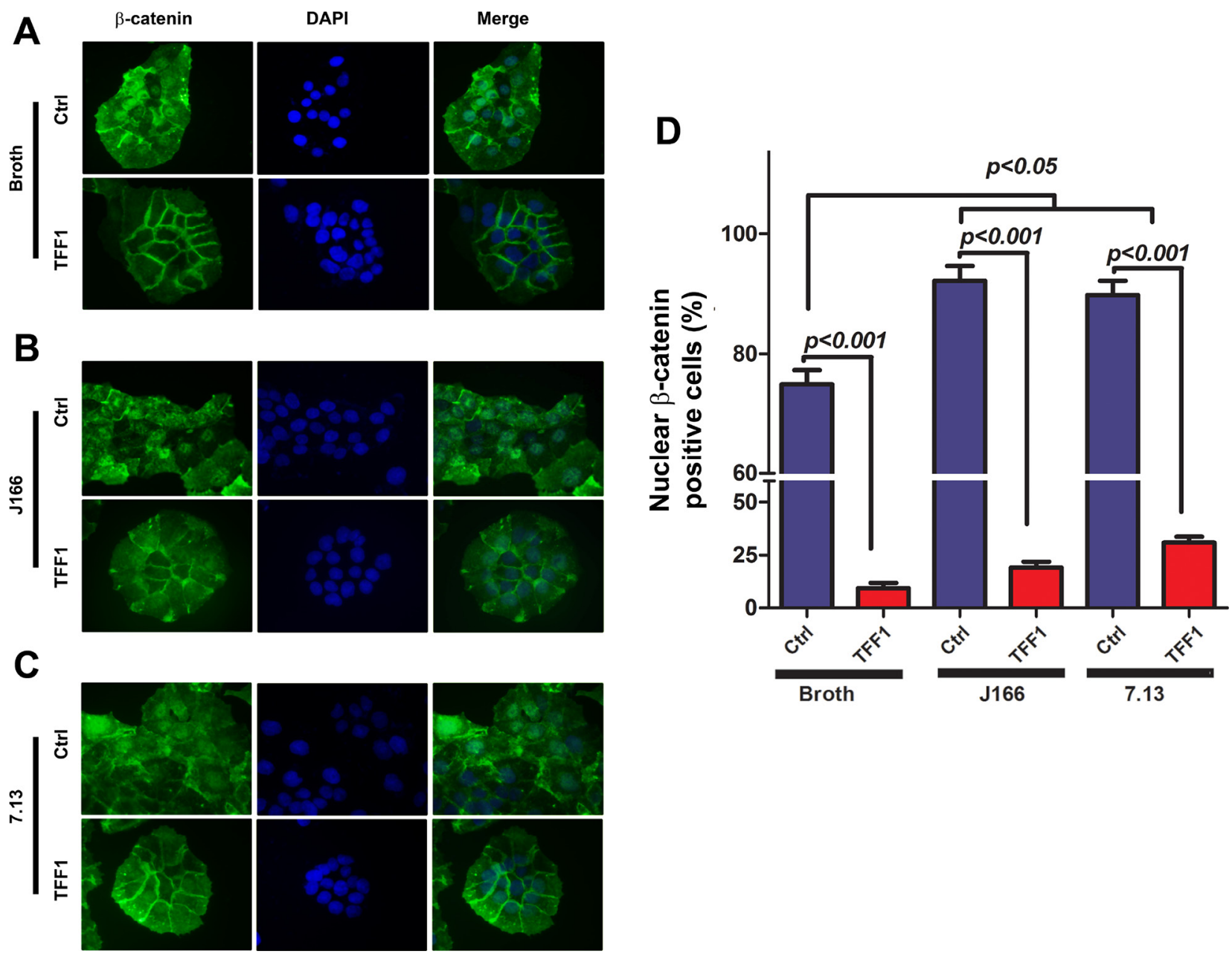

Figure 1: Reconstitution of TFF1 suppresses $\boldsymbol{H}$. pylori-mediated nuclear localization of $\boldsymbol{\beta}$-catenin. A-C. Immunofluorescence assay of $\beta$-catenin in control and TFF1-expressing MKN28 cells with and without infection with H. pylori. (A) Uninfected cells. (B) Cells infected with H. pylori strain J166. (C) Cell infected with H. pylori strain 7.13. Nuclear localization of $\beta$-catenin is depicted in green. DAPI (blue) was used as a nuclear counterstain. Original magnification, $\times 400$. D. Graph indicating the percentage of at least 200 counted cells that show $\beta$-catenin nuclear staining.

A

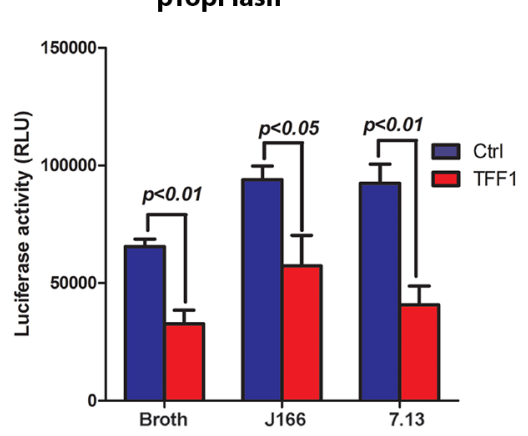

B

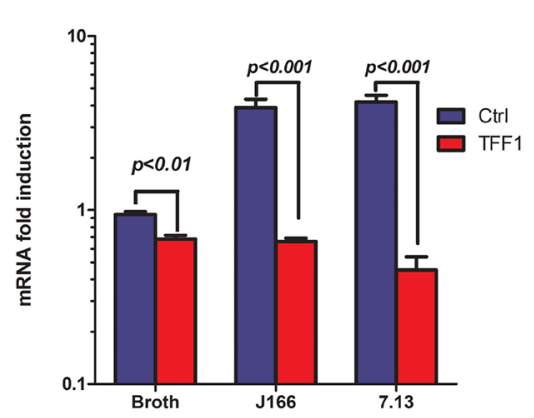

C

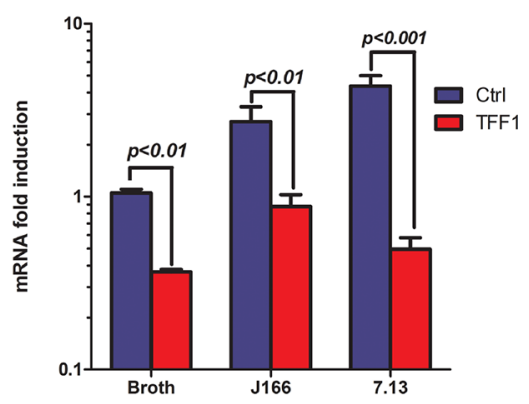

Figure 2: TFF1 abrogates $\mathrm{H}$. pylori-induced transcriptional activation of $\beta$-catenin and its target genes mRNA expression. A. pTopFlash luciferase reporter assays showing $\beta$-catenin and TCF-LEF transcriptional activity. TFF1 expressing cells showed a decrease of pTopFlash activity as compared to control cells after H. pylori infection. B-C. qRT-PCR data indicate mRNA downregulation of $\beta$-catenin target genes, $C C N D 1$ (B) and $c-M Y C$ (C) in MKN28 cells expressing TFF1 as compared to control cells after infection with $\mathrm{H}$. pylori $\mathrm{J} 166$ or 7.13 strains. These data are representative of at least three independent experiments. 


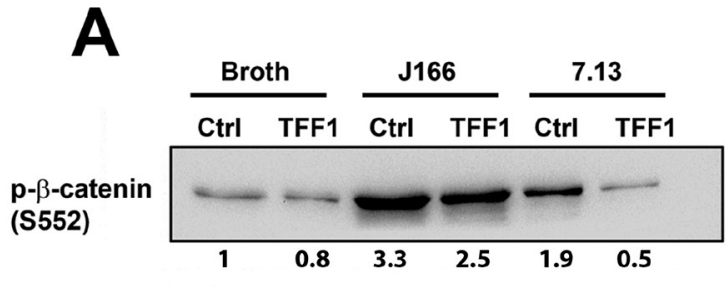

B

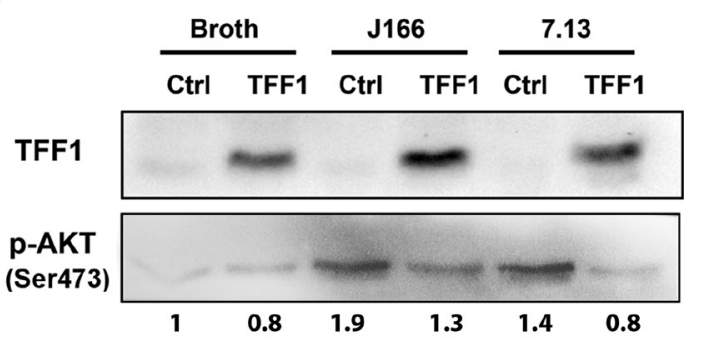

$\begin{array}{llllll}1 & 0.7 & 8.9 & 6.5 & 2.3 & 0.5\end{array}$

AKT

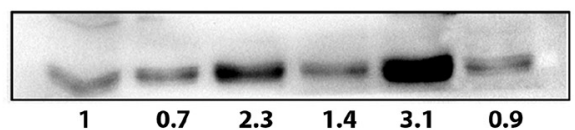

C-Myc

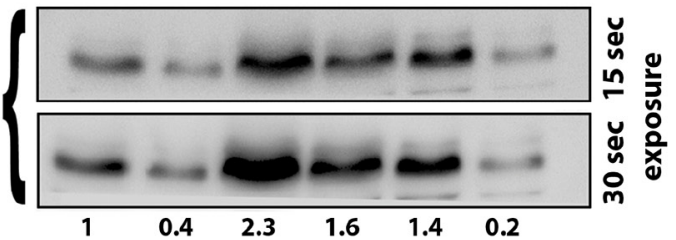

$\beta$-actin
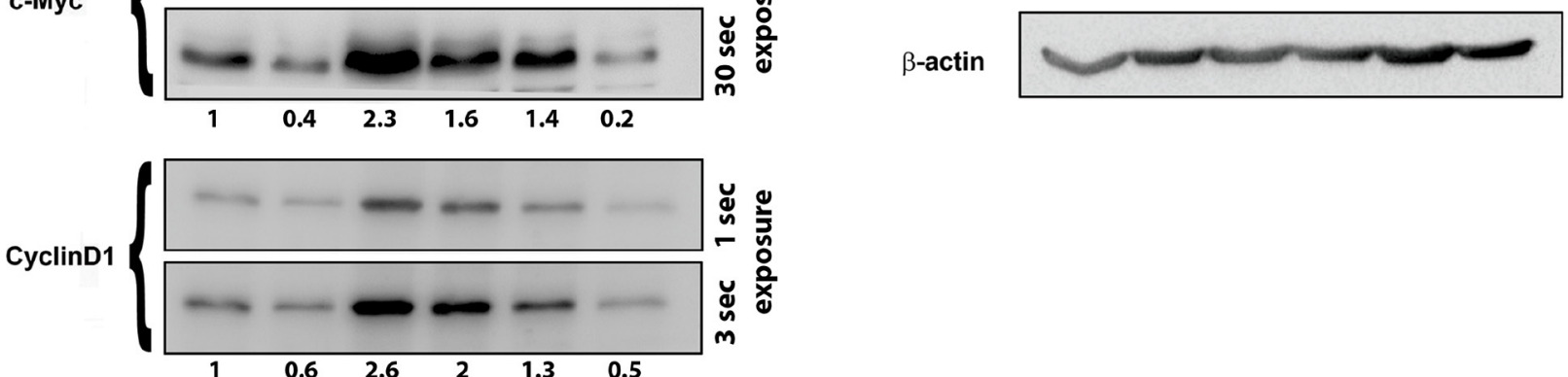

$\beta$-actin

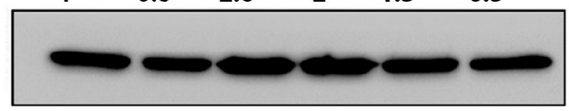

Figure 3: Reconstitution of TFF1 suppresses protein expression of $\beta$-catenin target genes in $\boldsymbol{H}$. pylori-infected cells. A-B. Western blot analysis of $\beta$-catenin downstream and upstream target genes. Reconstitution of TFF1 expression in H. pylori-infected MKN28 cells led to a decrease in protein levels of phospho- $\beta$-catenin (S552), $\beta$-catenin, c-MYC, and Cyclin D1 (A); phospho-AKT (Ser473) and AKT (B), as compared to their respective $H$. pylori-infected control cells. Protein loading was normalized for equal levels of $\beta$-actin. The intensity ratios of the indicated proteins level relative to Broth control were calculated after normalizing to $\beta$-actin using the ImageJ software $(\mathrm{NIH})$. The Western blot results are representative of three independent experiments.

\section{Loss of Tff1 promotes $H$. pylori-mediated activation of $\beta$-catenin and nuclear localization in gastric neoplasm mouse model}

To extend our in vitro investigation into an in vivo gastric neoplasm mouse model, Tff1-KO and Tff1-WT mice (6-8 weeks of age) were challenged with Brucella broth as uninfected controls or with $H$. pylori PMSS1 cagA+ strain. Immunohistochemistry analysis of the antropyloric region of the gastric tissue from Tff1-WT-infected mice showed a small but significant $(p<0.05)$ increase of nuclear $\beta$-catenin staining as compared to uninfected Tff1-WT mice (Figure 4A). However, in infected Tff1-KO mice, the immunostaining showed a significant $(p<0.001)$ increase of $\beta$-catenin nuclear localization as compared to uninfected Tff1-KO mice (Figure 4B). The quantification of the immunohistochemistry data demonstrated a significant increase of the percentage of $\beta$-catenin nuclear staining in uninfected Tffl-KO mice as compared to uninfected Tffl-WT mice (Figure 4C). However, in $H$. pylori-infected Tff1-KO mice, our analysis showed an even more significant $(p<0.001)$ increase of $\beta$-catenin nuclear staining as compared to uninfected Tffl-KO (Figure 4C). Next, we examined the mRNA expression of $\beta$-catenin downstream target genes in gastric epithelial cells from infected and uninfected mice. The qRT-PCR data confirmed the immunohistochemistry results and indicated a significant increase of $c-M y c$ and $C c n d 1$ mRNA expression in Tff1-KO as compared to Tffl-WT mice following $H$. pylori infection (Figure 5A-5B). The data also showed that $H$. pylori infection significantly increased $c-M y c$ and Ccndl mRNA levels in Tffl-KO mice as compared to uninfected mice (Figure 5A-5B). Collectively, these data clearly indicate that loss of Tff1 in addition to $H$. pylori infection increase the activation and nuclear localization of $\beta$-catenin in our Tff1-KO gastric cancer mouse model.

\section{H. pylori infection augments gastric cell proliferation and tumorigenesis in Tff1-KO mice}

Next, we investigated whether $H$. pylori infection in combination with Tff1 loss could further increase cell proliferation, a hallmark function of $\beta$-catenin activation, and would subsequently increase the rate of tumorigenesis. We subjected gastric tissues from Tff1-WT and Tffl-KO uninfected or H. pylori (PMSS1 strain)-infected mice similar in age to immunohistochemical staining of Ki-67. 
A

Tff1 WT

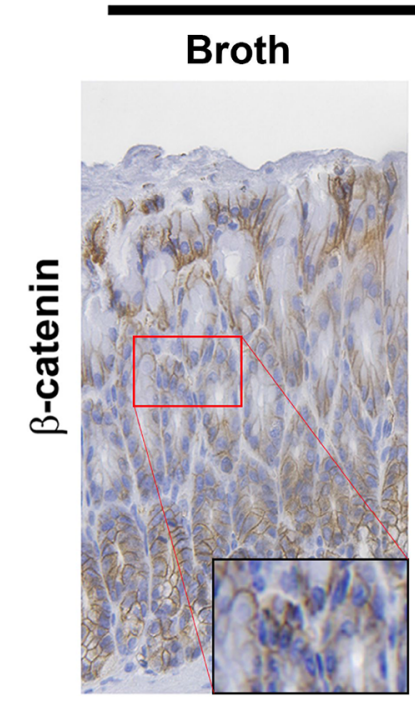

B Tff1 KO
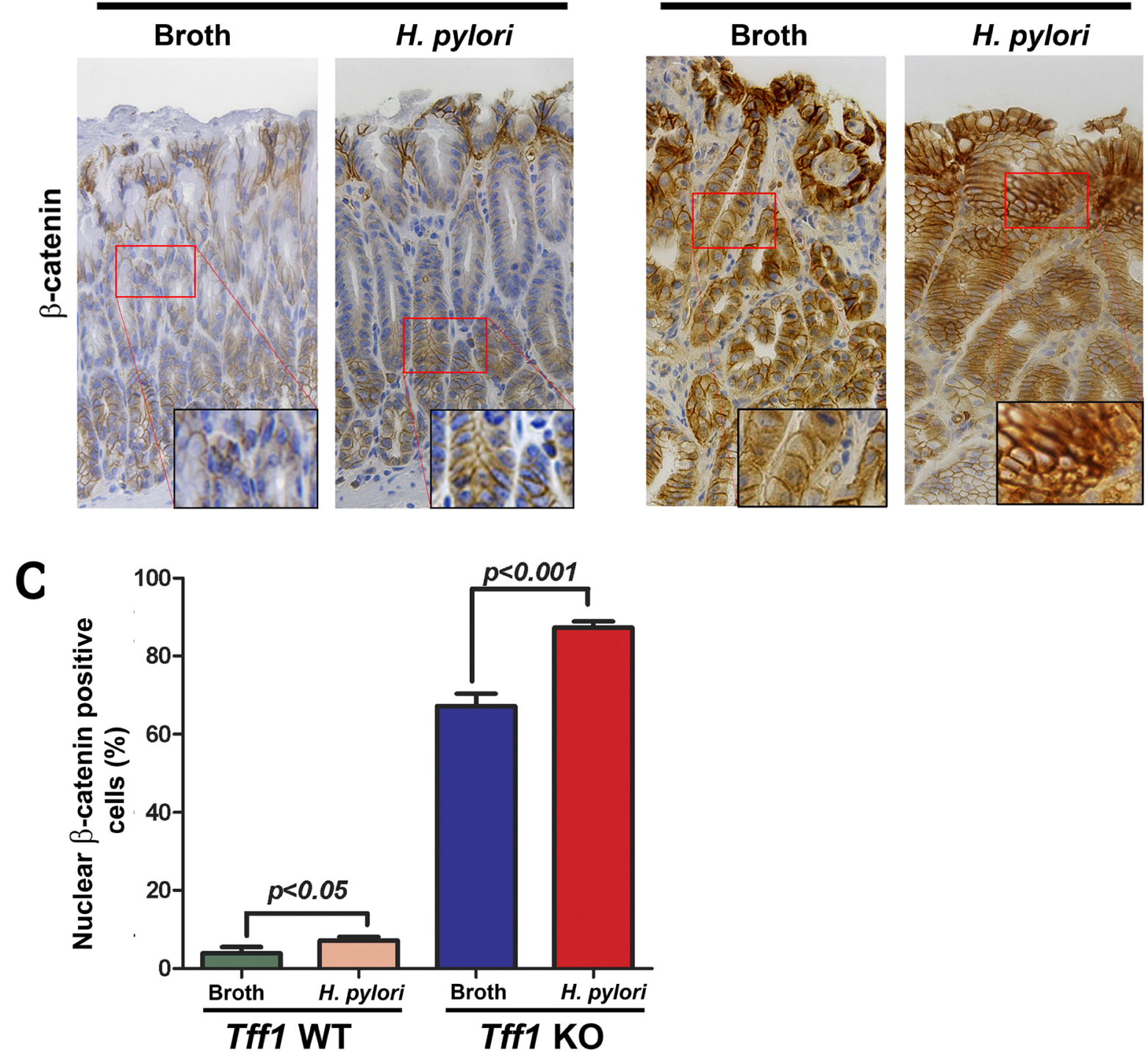

Figure 4: $\boldsymbol{H}$. pylori infection enhances nuclear accumulation of $\beta$-catenin in Tff1 knockout gastric cancer mouse model. A-B. Immunohistochemistry analysis of $\beta$-catenin expression in the antropyloric gastric mucosa from uninfected or $H$. pylori (PMSS1)-infected Tff1-wild-type (Tff1-WT) mice (9-10 months of age) (A) and Tff1-knockout (Tff1-KO) mice (9-10 months of age) (B), mice were challenged at 6-8 weeks of age with either sterile Brucella broth or H. pylori strain (PMSS1) by oral gavage. C. Quantification of nuclear $\beta$-catenin-positive staining in at least 200 counted cells presented as percentage \pm SEM is shown.

In the $H$. pylori-infected Tff1-WT mice, Ki-67 staining was observed in the basal proliferative region of the gastric glands similar to the uninfected Tff1-WT mice with a slight increase of the percentage of $\mathrm{Ki}-67$ positive nuclear staining (Figure 6A-6C, $p<0.05$ ). However, the gastric tissues in the infected Tffl-KO mice exhibited a significant increase $(p<0.001)$ of the percentage of positive nuclear Ki-67 staining as compared to uninfected Tff1-KO mice. Of note, the Ki-67 staining extended to the surface of the mucosa in both infected and uninfected Tff1KO mice (Figure 6B-6C).

Because the proliferation-associated marker Ki-67 is a characteristic of the antropyloric gastric lesions, we investigated the histological changes in uninfected and infected mice with $H$. pylori. Histological analysis using H\&E staining indicated a marked increase of invasive adenocarcinoma occurrence among infected Tff1-KO mice ( 7 out of $21,33 \%$ ) as compared to age-matched (8-15 month old mice) uninfected Tffl-KO mice (5 out of 48, 10\%), using our inventory from 2009-2014 (Figure 6D). However, both infected and uninfected Tffl-KO mice showed no significant difference in occurrence of LGD (62\% and $71 \%$ respectively) (Figure 6D). Conversely, Tff1-WT mice infected with $H$. pylori did not develop any dysplastic or invasive gastric lesions. Taken together, the data demonstrated that TFF1 is a negative regulator of 
A

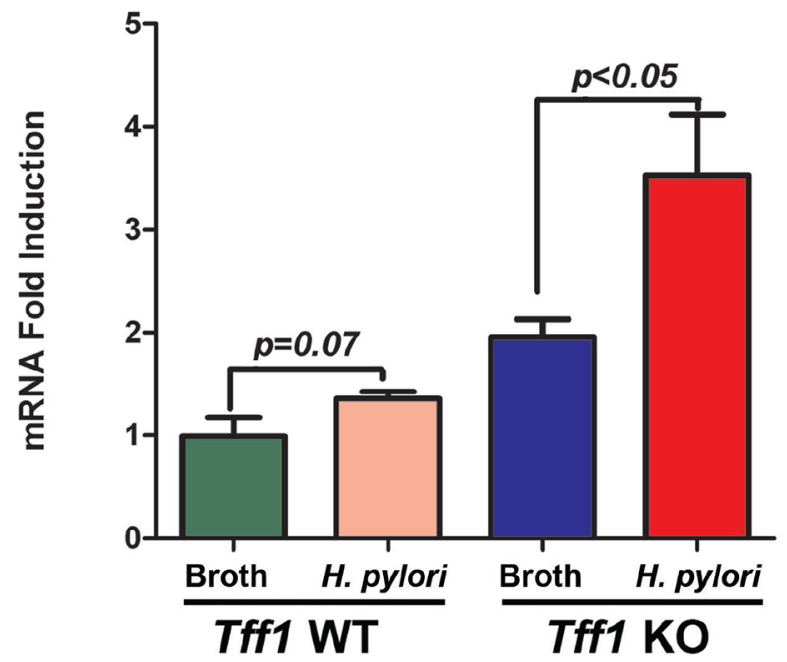

B

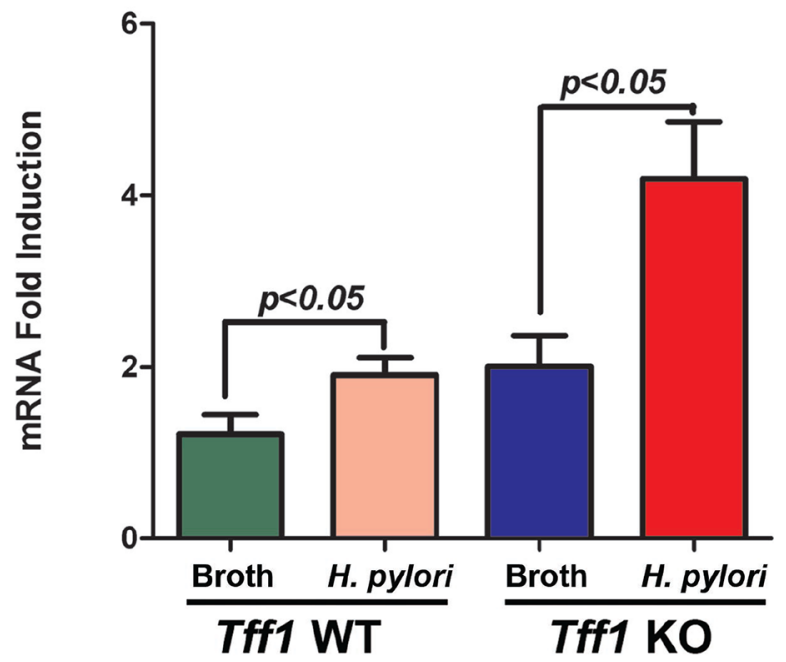

Figure 5: $\mathrm{H}$. pylori infection increases mRNA expression of $\beta$-catenin target genes in Tff1-knockout mice gastric tissues. A-B. Quantitative RT-PCR analysis showing mRNA expression levels of $\beta$-catenin target genes, $c$-Myc (A) and $C c n d 1$ (B) in uninfected or H. pylori strain (PMSS1) infected Tff1-WT and TffI-KO mice (12 weeks of age, 10 mice each).

H. pylori-induced $\beta$-catenin activation in vivo and in vitro. The loss of TFF1 promotes the progression of histological lesions in response to $H$. pylori infection.

\section{DISCUSSION}

Despite accumulating evidence from in vitro studies on the role of $H$. pylori in activating several oncogenic pathways, such as $\beta$-catenin [28-30], there is less information about the contribution of molecular events to H. pylori-mediated gastric tumorigenesis. Several studies have reported that long-term infection with $H$. pylori alone can induce chronic gastritis, chronic atrophic gastritis, and hyperplastic gastritis but is not sufficient to induce gastric cancer in wild-type mice $[24,31,32]$. We and others have demonstrated that silencing of $T F F 1$ promotes gastric tumorigenesis and cancer development in mouse and human $[4,5,7,33]$. In this report, we investigated the role of the TFF 1 in regulating $H$. pylori-mediated activation of $\beta$-catenin and gastric tumorigenesis. We provided evidence that TFF1 suppresses $H$. pylori-induced $\beta$-catenin activation in vitro and in vivo.

H. pylori plays an important role in activation of $\beta$-catenin through its oncoprotein $\mathrm{CagA}$; this activation is dependent on its translocation and nuclear accumulation [34-36]. In accordance with the reported data, our in vitro results demonstrated an increase of $\beta$-catenin activation, as indicated by its nuclear accumulation and the pTopFlash reporter data after $H$. pylori infection. In contrast, the reconstitution of TFF1 expression reduced $H$. pylori-induced $\beta$-catenin activation. The in vivo data showed that $H$. pylori infection of Tffl-WT mice induced a slight increase of $\beta$-catenin nuclear localization, indicating its activation in accordance with previously reported findings [34, 35]. However, in H. pylori-infected Tff1-KO mice, $\beta$-catenin was highly activated as indicated by the increased percentage of nuclear $\beta$-catenin positive gastric epithelial cells. While we have previously shown that TFF1 negatively regulates $\beta$-catenin [7], our present findings suggest that TFF1 plays an important role in overcoming and suppressing $H$. pylori-mediated activation of oncogenic $\beta$-catenin, where loss of Tff1 accelerated the progression towards invasive gastric adenocarcinoma in mice.

Increased activation and mutation of the $\beta$-catenin gene have been demonstrated in various cancers [37]. Notably, approximately $26 \%$ of gastric carcinomas have been reported to carry $\beta$-catenin activating mutations [38]. The expression of $\beta$-catenin target genes $c-M Y C$ and cyclin D1 (CCND1), which promote tumorigenesis, is increased within $H$. pylori colonized human gastric mucosa and during coculture of $H$. pylori with gastric epithelial cells in vitro $[26,39,40]$. Our data demonstrated that the reconstitution of TFF1 expression in gastric cancer cells abrogated $H$. pylori-induced activation of $\beta$-catenin and decreased mRNA and protein expression of c-myc and cyclin D1. Conversely, loss of Tff1 expression in $H$. pylori-infected mice significantly increased $c-M y c$ and Ccnd1 mRNA expression; this increase was significantly enhanced as compared to uninfected Tffl-KO mice. This confirms the suppressive effect of TFF1 on H. pyloriinduced activation of $\beta$-catenin in vivo and in vitro. These results suggest that $H$. pylori capitalizes on TFF1 loss to increase activation of $\beta$-catenin transcription activity and expression of oncogenes.

Colonization of gastric mucosa by $H$. pylori and deregulation of $\beta$-catenin signaling have been reported to induce epithelial hyperproliferation, which increase 

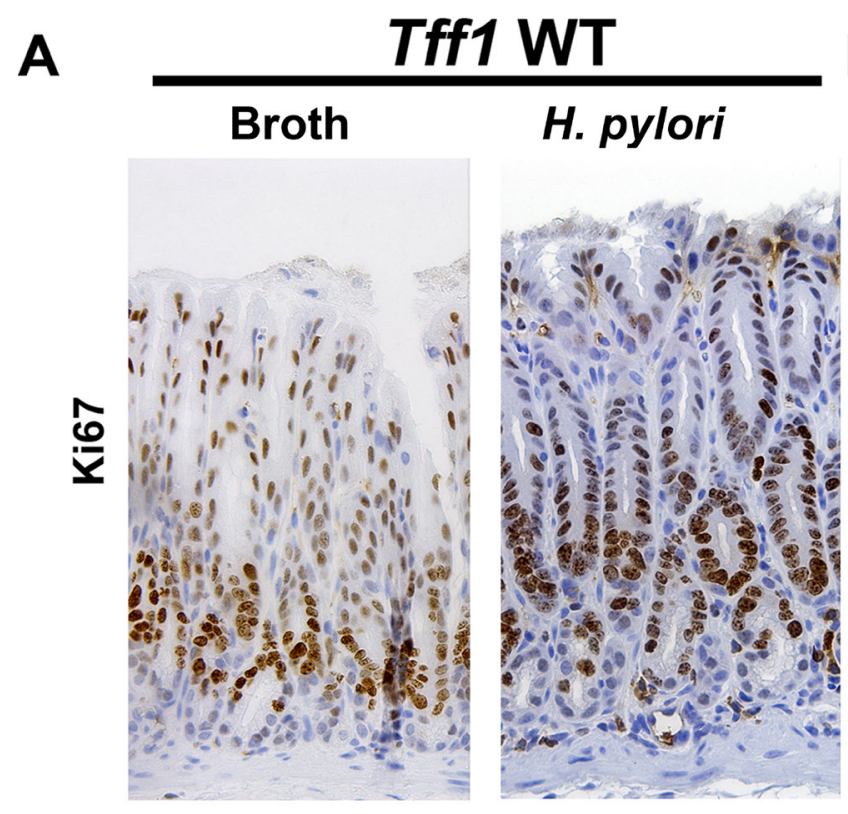

B Tff1 KO

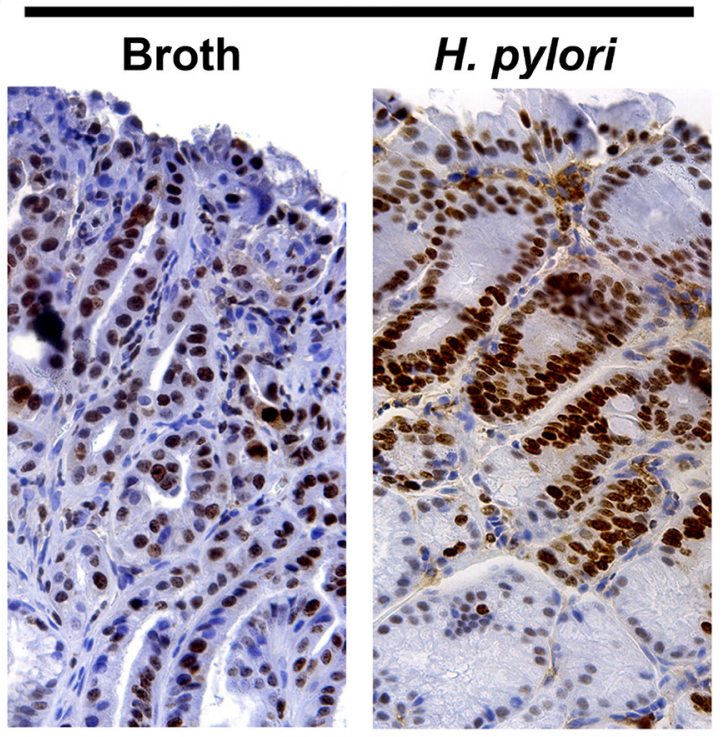

C
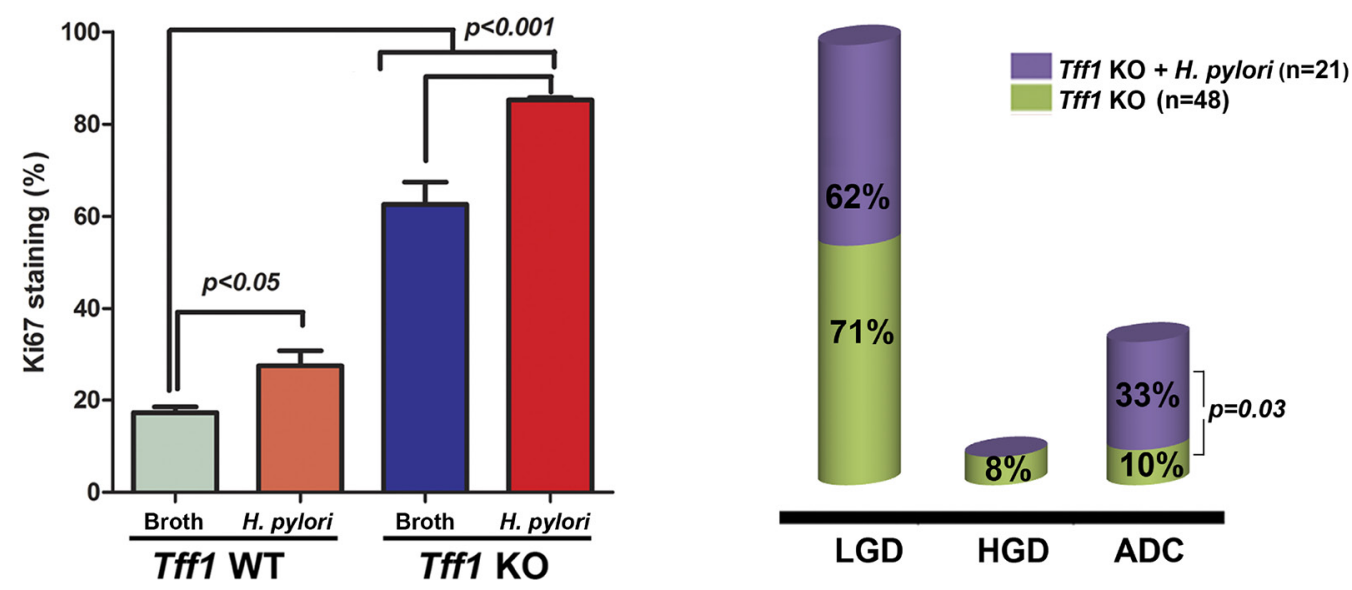

Figure 6: $\boldsymbol{H}$. pylori infection enhances gastric cell proliferation and tumorigenesis in Tff1-knockout mice. A-B. Representative images of Ki-67 immunostaining (brown nuclei) of serial sections, as in Figure 3, of gastric glands in Tff1-WT (9-10 months of age) (A) and Tffl-KO (9-10 Months of age) (B) uninfected or H. pylori-infected mice. Original magnification $(\times 100)$ was shown. C. The quantitative data of Ki-67 immunostaining in at least 200 counted cells were presented as percentage \pm SEM. D. A diagram showing the percentage of uninfected or infected TffI-KO mice with low-grade dysplasia (LGD), high-grade dysplasia (HGD), or invasive adenocarcinoma (ADC).

the risk for gastric adenocarcinoma [26, 41]. Our data demonstrated that $H$. pylori infection significantly enhances cell proliferation and the incidence of invasive gastric adenocarcinoma in the Tff1-KO mice. Interestingly, our results suggest that Tff1 plays a protective role in the stomach by suppressing $H$. pylori-induced activation of $\beta$-catenin. Based on our results, infection of Tffl-WT mice with $H$. pylori alone was not sufficient to drive gastric tumorigenesis, a finding that is consistent with epidemiological studies that demonstrate that almost half of the world's population is infected with $H$. pylori with less than $1 \%$ progressing to gastric cancer $[42,43]$. These data suggest that additional molecular hits are required to promote $H$. pylori-mediated gastric tumorigenesis; loss of Tffl is, therefore, plausibly a high-risk molecular event. Of note, approximately two-thirds of human gastric cancers, where $H$. pylori is a risk factor, demonstrate downregulation of TFF1 expression $[9,11,12]$. Our in vivo study demonstrated a significantly increased incidence of invasive adenocarcinoma in $\mathrm{H}$. pylori infected Tffl-KO mice as compared to uninfected Tff1-KO mice. However, only one-third of the $H$. pylori infected Tffl-KO mice developed invasive gastric adenocarcinoma. This finding may suggest that multiple $H$. pylori infections and/or a 
longer follow-up are needed to obtain a higher incidence of invasive gastric adenocarcinoma in the Tffl-KO mouse model.

In summary, our study demonstrates that TFF1 plays an essential protective role against $H$. pylorimediated oncogenic activation of $\beta$-catenin. The loss of TFF 1 could define a turning point towards activation of oncogenic $\beta$-catenin and expression of oncogenes in gastric tumorigenesis.

\section{MATERIALS \& METHODS}

\section{Ethics statement}

Procedures and experiments with mice were authorized by the Animal Care and Use Committee of the University of Vanderbilt and were carried out according to the guidelines.

\section{Cell culture and reagents}

Human gastric cancer MKN28 cells were obtained from ATCC (Manassas, VA). These cells were maintained in Dulbecco's modified Eagle's medium (DMEM; GIBCO, Carlsbad, CA) supplemented with 10\% fetal bovine serum (FBS; Invitrogen, Carlsbad, CA) and 1\% penicillin/ streptomycin (GIBCO). Cells were maintained at $37^{\circ} \mathrm{C}$ in an atmosphere containing 5\% CO2. Specific antibodies against phospho-AKT (Ser473), AKT, phospho- $\beta$-catenin (Ser552), $\beta$-catenin, Cyclin D1 and $\beta$-actin were purchased from Cell Signaling Technology (Beverly, MA). c-Myc antibody was obtained from Santa Cruz Biotechnology, Inc. (Santa Cruz, CA).

\section{Reconstitution of TFF1 expression}

To reconstitute the expression of TFF1 in MKN28 cells, we used an adenovirus expression system. We subcloned the TFF1 coding sequence from the pcDNA3.1/ TFF1 plasmid into the adenoviral shuttle vector (pACCMV). The recombinant adenovirus-expressing TFF1 was generated by co-transfecting HEK-293 cells with the shuttle and backbone adenoviral (pJM17) plasmids using the Calcium Phosphate Transfection kit (Applied Biological Materials, Richmond, BC). After infection, the MKN28 cells were analyzed for the expression of TFF1 by qRT-PCR.

\section{H. pylori bacterial strains and culture conditions}

For the in vitro studies, we used two CagA+ H. pylori carcinogenic strains, "J166" a clinical isolate of human-derived $H$. pylori, and "7.13" a rodent adapted strain derived from B128 H. pylori, which have been described previously [24, 44-47]. In the in vivo study, we used the wild-type rodent-adapted $\operatorname{cag} A+H$. pylori strain (PMSS1), a clinical isolate from a duodenal ulcer patient and the parental strain of the mousederivative Sydney strain 1 (SS1) [48]. All H. pylori plate cultures were performed on Brucella agar (BBL/Becton Dickinson, Sparks, MD) supplemented with 5\% heatinactivated newborn calf serum (Invitrogen) and ABPNV (amphotericin B, $20 \mathrm{mg} /$ liter; bacitracin, $200 \mathrm{mg} / \mathrm{liter}$; polymyxin B, $3.3 \mathrm{mg} /$ liter; nalidixic acid, $10.7 \mathrm{mg} / \mathrm{liter}$; vancomycin, $100 \mathrm{mg} /$ liter) antibiotics (all from SigmaAldrich, Milwaukee, WI). H. pylori MPSS1 strain liquid cultures for mouse inoculation were grown in Brucella broth (BD Biosciences) with 5\% NCS and antibiotic supplementation for approximately $24 \mathrm{~h}$, pelleted by centrifugation, and suspended in Brucella broth.

\section{Immunofluorescence assay}

MKN28 cells infected with control or TFF1 adenoviruses (10 MOI) were plated in 8-well chambers. After 48 h, cells were infected with either $H$. pylori J166 or 7.13 strains $(100: 1)$ for $3 \mathrm{~h}$. Cells were washed with PBS and fixed with fresh 4\% paraformaldehyde solution for $15 \mathrm{~min}$ at room temperature. Cells were then washed twice with PBS, followed by incubation in $10 \%$ normal goat serum blocking solution (Zymed Laboratories, San Francisco, CA) for $20 \mathrm{~min}$ at room temperature in a humidified chamber. Cells were incubated in the specific primary antibody against $\beta$-catenin diluted in PBS $(1: 400)$ for $2 \mathrm{~h}$ at room temperature in a humidified chamber. Cells were washed 3 times in PBS and incubated in fluorescein isothiocyanate (FITC)-tagged secondary antibody $(1: 1,000$; Jackson Immunoresearch, West Grove, PA) for $45 \mathrm{~min}$ at room temperature in a humidified chamber. The cells were then washed in PBS, mounted with Vectashield/DAPI (Vector Laboratories, Burlingame, CA), and visualized using an Olympus BX51 fluorescence microscope (Olympus Co. Center Valley, PA). For quantification, ImageJ software (US National Institutes of Health) was used. The images were transformed into 8-bit and a region of interest (ROI) was randomly selected in the nucleus and cytoplasm. The ratio of integrated density in the nucleus versus cytoplasm was determined by measuring the intensity density of the ROI in the nucleus and cytoplasm. The percentage of cells that show $\beta$-catenin nuclear staining was determined based on the value of the density ratio; $a$ value equal to or less than 1 was considered negative, $a$ value more than 1 was considered positive.

\section{Luciferase reporter assay}

To evaluate the transcriptional activity of TCF/ $\beta$-catenin, we used the luciferase reporter construct, pTopFlash, with six TCF binding sites (Upstate Biotechnology, Waltham, MA). Fugene 6 was used for transfection as directed by the manufacturer's protocol (Roche Applied Science, Indianapolis, IN). After infection with TFF1 adenoviral particles (10 MOI) or 
control adenoviruses for $3 \mathrm{~h}$, cells were transfected with pTopFlash. $48 \mathrm{~h}$ later, cells were infected with H. pylori strains J166 or 7.13 (100:1), and luciferase and $\beta$-galactosidase activity were measured as described previously [49]. The firefly luciferase activity was normalized to $\beta$-galactosidase activity and expressed as relative luciferase unit with \pm standard error of the mean (SEM).

\section{Quantitative real-time RT-PCR}

Total RNA was isolated using the RNeasy Mini kit (Qiagen, Germantown, MD) and cDNA synthesis was performed using an iScript cDNA Synthesis Kit (Bio-Rad, Hercules, CA). Primers specific for mouse and human genes were designed using the online software Primer 3 (http://frodo.wi.mit.edu/primer3/). The forward and reverse primers were designed to span two different exons for each gene (human: $c-M Y C$ and $C C N D 1$; mouse: $c-M y c$ and $C$ cnd1), as previously described [7]. All primers were purchased from Integrated DNA Technologies (IDT, Coralville, IA). The qRT-PCR was performed using an iCycler (Bio-Rad), with the threshold cycle number determined by use of iCycler software version 3.0. Reactions were performed in triplicate and the threshold cycle numbers were averaged. The results of the genes were normalized to housekeeping genes, $18 \mathrm{~S}$ for human and actin for mouse. Expression ratios were calculated according to the formula $2^{(\mathrm{Rt}-\mathrm{Et})} / 2^{(\mathrm{Rn}-\mathrm{En})}[50]$ where $\mathrm{Rt}$ is the threshold cycle number for the reference gene observed in the test samples; Et is the threshold cycle number for the experimental gene observed in the test samples, $\mathrm{Rn}$ is the threshold cycle number for the reference gene observed in the reference samples, and En is the threshold cycle for the experimental gene observed in the reference samples. $\mathrm{Rn}$ and En values were calculated as an average of all reference samples.

\section{Western blotting}

Cell lysates were prepared in RIPA buffer containing Halt Protease and Phosphatase Inhibitor Cocktail (Pierce Biotechnology, Inc., Rockford, IL) and were centrifuged at $4,390 \mathrm{~g}$ for $10 \mathrm{~min}$ at $4^{\circ} \mathrm{C}$. Protein concentration was measured using a Bio-Rad Protein Assay (Bio-Rad). Equal amounts of proteins $(10-15 \mu \mathrm{g})$ from each sample were subjected to SDS/PAGE and transferred onto nitrocellulose membranes. Target proteins were detected by using specific antibodies.

\section{Animals infection}

Tff1-KO mouse model of gastric tumorigenesis [3] and normal Tff1-WT mice, at 6-8 weeks of age, were inoculated by oral gavage with $1 \times 10^{9}$ colony-forming units of $H$. pylori MPSS1 strain in $0.1 \mathrm{ml}$ of Brucella broth or Brucella broth alone as a vehicle control
[51]. Mice were euthanized at 4, 24, 32, and 48 weeks post-challenge (8-10 mice per group). The 4-week challenged mice, with non-dysplastic lesions, were used for qRT-PCR to detect early changes in the expression of $\beta$-catenin target genes; older mice groups were used for histological evaluation of progression. Frozen and formalin fixed paraffin-embedded stomach tissue samples were collected from $H$. pylori-infected and uninfected Tff1-KO and Tff1-WT mice. All procedures were approved by the Animal Care Committee of Vanderbilt University.

\section{Histologic evaluation and immunohistochemical assessment}

Histopathological classification and grading of the gastric tissues were performed by our pathologists (MBP and MKW) on H\&E stained sections. Immunohistochemistry analysis for $\beta$-catenin was performed on paraffin-embedded stomach tissues using $\beta$-catenin mouse monoclonal antibody (Cell Signaling). Serial sections of the same embedded tissues were used to detect proliferating cells with a mouse monoclonal antibody directed against the Ki-67 antigen (Sigma-Aldrich).

\section{Statistical analysis}

Using GraphPad Prism software, a One-way ANOVA Newman-Keuls Multiple Comparisons Test was used to compare the differences between three or more groups, and a two-tailed Student's test was used to compare the statistical difference between two groups. The differences were considered statistically significant when the $P$ value was $<0.05$.

\section{ACKNOWLEDGMENTS}

This study was supported by grants from the Department of Veterans Affairs (WER), Vanderbilt SPORE in Gastrointestinal Cancer (P50 CA95103), Vanderbilt Ingram Cancer Center (P30 CA68485), and the Vanderbilt Digestive Disease Research Center (DK058404). The contents of this work are solely the responsibility of the authors and do not necessarily represent the official views of the Department of Veterans Affairs, National Institutes of Health, or Vanderbilt University.

\section{CONFLICTS OF INTEREST}

All authors indicated "no conflict of interest".

\section{SUMMARY}

Because of the frequent loss of TFF1 in gastric cancer, we investigated its impact on $\beta$-catenin activation and gastric tumorigenesis in response to $H$. pylori 
infection. The loss of TFF1 promoted $H$. pylori-mediated oncogenic activation of $\beta$-catenin and cell proliferation, hallmarks of gastric carcinogenesis.

\section{REFERENCES}

1. Tomasetto C, Masson R, Linares JL, Wendling C, Lefebvre O, Chenard MP, Rio MC. pS2/TFF1 interacts directly with the VWFC cysteine-rich domains of mucins. Gastroenterology. 2000; 118:70-80.

2. Tanaka T, Nakamura J, Kitajima Y, Kai K, Miyake S, Hiraki M, Ide T, Koga Y, Noshiro H. Loss of trefoil factor 1 is regulated by DNA methylation and is an independent predictive factor for poor survival in advanced gastric cancer. Int J Oncol. 2013; 42:894-902.

3. Lefebvre O, Chenard MP, Masson R, Linares J, Dierich A, LeMeur M, Wendling C, Tomasetto C, Chambon P, Rio MC. Gastric mucosa abnormalities and tumorigenesis in mice lacking the pS2 trefoil protein. Science. 1996; 274:259-262.

4. Buache E, Etique N, Alpy F, Stoll I, Muckensturm M, Reina-San-Martin B, Chenard MP, Tomasetto C, Rio MC. Deficiency in trefoil factor 1 (TFF1) increases tumorigenicity of human breast cancer cells and mammary tumor development in TFF1-knockout mice. Oncogene. 2011; 30:3261-3273.

5. Soutto M, Belkhiri A, Piazuelo MB, Schneider BG, Peng D, Jiang A, Washington MK, Kokoye Y, Crowe SE, Zaika A, Correa P, Peek RM Jr., El-Rifai W. Loss of TFF1 is associated with activation of NF-kappaB-mediated inflammation and gastric neoplasia in mice and humans. J Clin Invest. 2011; 121:1753-1767.

6. Katsha A, Soutto M, Sehdev V, Peng D, Washington MK, Piazuelo MB, Tantawy MN, Manning HC, Lu P, Shyr Y, Ecsedy J, Belkhiri A, El-Rifai W. Aurora kinase A promotes inflammation and tumorigenesis in mice and human gastric neoplasia. Gastroenterology. 2013; 145:1312-1322. e1311-1318.

7. Soutto M, Peng D, Katsha A, Chen Z, Piazuelo MB, Washington MK, Belkhiri A, Correa P, El-Rifai W. Activation of beta-catenin signalling by TFF1 loss promotes cell proliferation and gastric tumorigenesis. Gut. 2014; .

8. Park WS, Oh RR, Park JY, Lee JH, Shin MS, Kim HS, Lee HK, Kim YS, Kim SY, Lee SH, Yoo NJ, Lee JY. Somatic mutations of the trefoil factor family 1 gene in gastric cancer. Gastroenterology. 2000; 119:691-698.

9. Carvalho R, Kayademir T, Soares P, Canedo P, Sousa S, Oliveira C, Leistenschneider P, Seruca R, Gott P, Blin N, Carneiro F, Machado JC. Loss of heterozygosity and promoter methylation, but not mutation, may underlie loss of TFF1 in gastric carcinoma. Lab Invest. 2002; 82:1319-1326.

10. Tomita H, Takaishi S, Menheniott TR, Yang X, Shibata W, Jin G, Betz KS, Kawakami K, Minamoto T, Tomasetto C,
Rio MC, Lerkowit N, Varro A, Giraud AS, Wang TC. Inhibition of gastric carcinogenesis by the hormone, gastrin, is mediated by suppression of TFF1 epigenetic silencing. Gastroenterology. 2010; .

11. McChesney PA, Aiyar SE, Lee OJ, Zaika A, Moskaluk C, Li R, El-Rifai W. Cofactor of BRCA1: a novel transcription factor regulator in upper gastrointestinal adenocarcinomas. Cancer Res. 2006; 66:1346-1353.

12. Im S, Yoo C, Jung JH, Choi HJ, Yoo J, Kang CS. Reduced expression of TFF1 and increased expression of TFF3 in gastric cancer: correlation with clinicopathological parameters and prognosis. Int J Med Sci. 2013; 10:133-140.

13. Clevers H, Nusse R. Wnt/beta-catenin signaling and disease. Cell. 2012; 149:1192-1205.

14. Zhang HF, Xue YW. Wnt pathway is involved in advanced gastric carcinoma. Hepato-Gastroenterol. 2008; 55:1126-1130.

15. Polakis P. Wnt signaling and cancer. Genes \& development. 2000; 14:1837-1851.

16. Li L, Ji SY, Yang JL, Li XX, Zhang J, Zhang Y, Hu ZY, Liu YX. Wnt/beta-catenin signaling regulates follicular development by modulating the expression of Foxo3a signaling components. Mol Cell Endocrinol. 2014; 382:915-925.

17. Yang Y, Yang JJ, Tao H, Jin WS. New perspectives on beta-catenin control of cell fate and proliferation in colon cancer. Food Chem Toxicol. 2014; 74:14-19.

18. Marshall BJ, Warren JR. Unidentified curved bacilli in the stomach of patients with gastritis and peptic ulceration. Lancet. 1984; 1:1311-1315.

19. Nomura A, Stemmermann G, Chyou P, Kato I, PerezPerez G, Blaser M. Helicobacter pylori infection and gastric carcinoma among Japanese Americans in Hawaii. N Engl J Med. 1991; 325:1132-1136.

20. Parsonnet J, Friedman G, Vandersteen D. Helicobacter pylori infection and the risk of gastric carcinoma. N Engl J Med. 1991; 325:1127-1131.

21. Matysiak-Budnik T, Laszewicz W, Lamarque D, Chaussade S. Helicobacter pylori and non-malignant diseases. Helicobacter. 2006; 11:27-31. Suppl 1.

22. Oliveira MJ, Costa AM, Costa AC, Ferreira RM, Sampaio P, Machado JC, Seruca R, Mareel M, Figueiredo C. CagA associates with c-Met, E-cadherin, and p120-catenin in a multiproteic complex that suppresses Helicobacter pyloriinduced cell-invasive phenotype. The Journal of infectious diseases. 2009; 200:745-755.

23. Hatakeyama M. Oncogenic mechanisms of the Helicobacter pylori CagA protein. Nature reviews Cancer. 2004; 4:688-694.

24. Franco AT, Israel DA, Washington MK, Krishna U, Fox JG, Rogers AB, Neish AS, Collier-Hyams L, Perez-Perez GI, Hatakeyama M, Whitehead R, Gaus K, O'Brie DP, Romero-Gallo J, Peek RM Jr. Activation of beta-catenin by carcinogenic Helicobacter pylori. Proc Natl Acad Sci U S A. $2005 ; 102: 10646-10651$. 
25. Bebb JR, Leach L, Zaitoun A, Hand N, Letley DP, Thomas R, Atherton JC. Effects of Helicobacter pylori on the cadherin-catenin complex. Journal of clinical pathology. 2006; 59:1261-1266.

26. Murata-Kamiya N, Kurashima Y, Teishikata Y, Yamahashi Y, Saito Y, Higashi H, Aburatani H, Akiyama T, Peek RM Jr., Azuma T, Hatakeyama M. Helicobacter pylori CagA interacts with E-cadherin and deregulates the betacatenin signal that promotes intestinal transdifferentiation in gastric epithelial cells. Oncogene. 2007; 26:4617-4626.

27. Guang W, Czinn SJ, Blanchard TG, Kim KC, Lillehoj EP. Genetic regulation of MUC1 expression by Helicobacter pylori in gastric cancer cells. Biochemical and biophysical research communications. 2014; 445:145-150.

28. Boltin D, Niv Y. Mucins in Gastric Cancer - An Update. Journal of gastrointestinal \& digestive system. 2013; 3:15519.

29. Micu G, Staniceanu F, Zurac S, Bastian A, Gramada E, Popp C, Andrei R, Tudorica L, Slavnea A, Olariu M, Tebeica T, Ene A, Mateescu R, Rimbas M, Voiosu R. E-cadherin and beta-catenin expression in gastric neoplastic and non-neoplastic lesions - correlations with H. pylori infection. Rom J Intern Med. 2010; 48:271-280.

30. Gnad T, Feoktistova M, Leverkus M, Lendeckel U, Naumann M. Helicobacter pylori-induced activation of beta-catenin involves low density lipoprotein receptorrelated protein 6 and Dishevelled. Mol Cancer. 2010; 9:31.

31. Hatakeyama M. Helicobacter pylori and gastric carcinogenesis. J Gastroenterol. 2009; 44:239-248.

32. Kim IJ, Park JH, Kang HC, Shin Y, Lim SB, Ku JL, Yang HK, Lee KU, Park JG. A novel germline mutation in the MET extracellular domain in a Korean patient with the diffuse type of familial gastric cancer. J Med Genet. 2003; 40:e97.

33. Soutto M, Chen Z, Saleh MA, Katsha A, Zhu S, Zaika A, Belkhiri A, El-Rifai W. TFF1 activates p53 through downregulation of miR-04 in gastric cancer. Oncotarget. 2014; 5:5663-5673.

34. Wroblewski LE, Piazuelo MB, Chaturvedi R, Schumacher M, Aihara E, Feng R, Noto JM, Delgado A, Israel DA, Zavros Y, Montrose MH, Shroyer N, Correa P, Wilson KT, Peek RM Jr. Helicobacter pylori targets cancerassociated apical-junctional constituents in gastroids and gastric epithelial cells. Gut. 2014;

35. Lee DG, Kim HS, Lee YS, Kim S, Cha SY, Ota I, Kim NH, Cha YH, Yang DH, Lee Y, Park GJ, Yook JI, Lee YC. Helicobacter pylori CagA promotes Snail-mediated epithelial-mesenchymal transition by reducing GSK-3 activity. Nat Commun. 2014; 5:4423.

36. Amieva MR, Vogelmann R, Covacci A, Tompkins LS, Nelson WJ, Falkow S. Disruption of the epithelial apicaljunctional complex by Helicobacter pylori CagA. Science. 2003; 300:1430-1434.
37. Ebert MP, Fei G, Kahmann S, Muller O, Yu J, Sung JJ, Malfertheiner P. Increased beta-catenin mRNA levels and mutational alterations of the APC and beta-catenin gene are present in intestinal-type gastric cancer. Carcinogenesis. 2002; 23:87-91.

38. Clements WM, Wang J, Sarnaik A, Kim OJ, MacDonald J, Fenoglio-Preiser C, Groden J, Lowy AM. beta-Catenin mutation is a frequent cause of Wnt pathway activation in gastric cancer. Cancer Res. 2002; 62:3503-3506.

39. Crawford HC, Krishna US, Israel DA, Matrisian LM, Washington MK, Peek RM Jr. Helicobacter pylori strainselective induction of matrix metalloproteinase- 7 in vitro and within gastric mucosa. Gastroenterology. 2003; 125:1125-1136.

40. Romano M, Ricci V, Di Popolo A, Sommi P, Del Vecchio Blanco C, Bruni CB, Ventura U, Cover TL, Blaser MJ, Coffey RJ, Zarrilli R. Helicobacter pylori upregulates expression of epidermal growth factor-related peptides, but inhibits their proliferative effect in MKN 28 gastric mucosal cells. J Clin Invest. 1998; 101:1604-1613.

41. Nagy TA, Wroblewski LE, Wang D, Piazuelo MB, Delgado A, Romero-Gallo J, Noto J, Israel DA, Ogden SR, Correa P, Cover TL, Peek RM Jr. beta-Catenin and p120 mediate PPARdelta-dependent proliferation induced by Helicobacter pylori in human and rodent epithelia. Gastroenterology. 2011; 141:553-564.

42. Peek RM Jr., Blaser MJ. Helicobacter pylori and gastrointestinal tract adenocarcinomas. Nature reviews Cancer. $2002 ; 2: 28-37$.

43. Imrie $\mathrm{C}$, Rowland $\mathrm{M}$, Bourke $\mathrm{B}$, Drumm B. Is Helicobacter pylori infection in childhood a risk factor for gastric cancer? Pediatrics. 2001; 107:373-380.

44. Styer CM, Hansen LM, Cooke CL, Gundersen AM, Choi SS, Berg DE, Benghezal M, Marshall BJ, Peek RM Jr., Boren T, Solnick JV. Expression of the BabA adhesin during experimental infection with Helicobacter pylori. Infect Immun. 2010; 78:1593-1600.

45. Solnick JV, Hansen LM, Salama NR, Boonjakuakul JK, Syvanen M. Modification of Helicobacter pylori outer membrane protein expression during experimental infection of rhesus macaques. Proc Natl Acad Sci U S A. 2004; 101:2106-2111.

46. Alm RA, Ling LS, Moir DT, King BL, Brown ED, Doig PC, Smith DR, Noonan B, Guild BC, deJonge BL, Carmel G, Tummino PJ, Caruso A, Uria-Nickelsen M, Mills DM, Ives C, et al. Genomic-sequence comparison of two unrelated isolates of the human gastric pathogen Helicobacter pylori. Nature. 1999; 397:176-180.

47. Franco AT, Johnston E, Krishna U, Yamaoka Y, Israel DA, Nagy TA, Wroblewski LE, Piazuelo MB, Correa P, Peek RM Jr. Regulation of gastric carcinogenesis by Helicobacter pylori virulence factors. Cancer Res. 2008; 68:379-387. 
48. Arnold IC, Lee JY, Amieva MR, Roers A, Flavell RA, Sparwasser T, Muller A. Tolerance rather than immunity protects from Helicobacter pylori-induced gastric preneoplasia. Gastroenterology. 2011; 140:199-209.

49. Smale ST. Beta-galactosidase assay. Cold Spring Harbor protocols. 2010; 2010. pdbprot5423.

50. El-Rifai W, Powell S. Molecular and biologic basis of upper gastrointestinal malignancy gastric carcinoma.
Surgical Oncology Clinics of North America. 2002; 11:273-291.

51. Fox JG, Wang TC, Rogers AB, Poutahidis T, Ge Z, Taylor N, Dangler CA, Israel DA, Krishna U, Gaus K, Peek RM Jr. Host and microbial constituents influence Helicobacter pylori-induced cancer in a murine model of hypergastrinemia. Gastroenterology. 2003; 124:1879-1890. 